University of Wollongong

Research Online

Australian Institute for Innovative Materials -

Papers

Australian Institute for Innovative Materials

$1-1-2018$

Carbon hollow nanobubbles on porous carbon nanofibers: An ideal host for high-performance sodium-sulfur batteries and hydrogen storage

\author{
Guanglin Xia \\ University of Wollongong, gx168@uowmail.edu.au \\ Lijun Zhang \\ Fudan University \\ Xiaowei Chen \\ Tsinghua University, Collaborative Innovation Center Of Quantum Matter, Chinese Academy Of Sciences, \\ Fudan University, Nanjing University, Jimei University, chenx@nju.edu.cn \\ Yuqin Huang \\ University of Wollongong, yh659@uowmail.edu.au \\ Dalin Sun \\ Fudan University, Max Planck Institute
}

See next page for additional authors

Follow this and additional works at: https://ro.uow.edu.au/aiimpapers

Part of the Engineering Commons, and the Physical Sciences and Mathematics Commons

Research Online is the open access institutional repository for the University of Wollongong. For further information contact the UOW Library: research-pubs@uow.edu.au 


\title{
Carbon hollow nanobubbles on porous carbon nanofibers: An ideal host for high- performance sodium-sulfur batteries and hydrogen storage
}

\author{
Abstract \\ One-dimensional (1D) carbon nanostructures have been intensively investigated because of their \\ intriguing features and great potential for practical application in various fields. This paper reports the \\ controllable fabrication of carbon hollow nanobubbles on porous carbon nanofibers (CHNBs@PCNFs) \\ through a general electrospinning strategy, with metal azides serving as both a bubbling and a porogen \\ reagent. The strong repulsive forces resulting from the intense release of $\mathrm{N} 2$ from the decomposition of \\ metal azides upon carbonization leads to the uniform formation of porous carbon nanofibers (PCNFs), \\ which could be facile tuned by heating rates and the amount of the bubbling reagent, simultaneously \\ constructed with carbon hollow nanobubbles (CHNBs) on the surface. Density functional theory \\ calculations reveal the strong interactions between terminal $\mathrm{Na}$ atoms in sodium polysulfides and $\mathrm{N}$ and \\ 0 atoms doped into CHNBs@PCNFs, which could effectively alleviate the shuttle effect of Na-S batteries \\ via adsorbing and trapping polysulfides. With strong adsorption capability of sodium polysulfides and \\ high electrical conductivity, these CHNBs@PCNFs are demonstrated to be an ideal sulfur host in room- \\ temperature sodium-sulfur batteries, which delivers a high reversible capacity of $256 \mathrm{~mA} \mathrm{~h} \mathrm{~g}-1$ (specific \\ energy density $384 \mathrm{~W} \mathrm{~h} \mathrm{kg-1}$ ) with a low decay rate of $0.044 \%$ per cycle at $2 \mathrm{C}$-rate. When \\ CHNBs@PCNFs used as functional supports for $\mathrm{MgH} 2$ nanoparticles, a significantly enhanced hydrogen \\ storage performance was achieved. The present work represents a critically important step in advancing \\ the electrospinning technique for generating 1D carbon nanostructures in a facile and universal manner.

\section{Disciplines} \\ Engineering | Physical Sciences and Mathematics

\section{Publication Details} \\ Xia, G., Zhang, L., Chen, X., Huang, Y., Sun, D., Fang, F., Guo, Z. \& Yu, X. (2018). Carbon hollow nanobubbles \\ on porous carbon nanofibers: An ideal host for high-performance sodium-sulfur batteries and hydrogen \\ storage. Energy Storage Materials, 14 314-323.

\section{Authors} \\ Guanglin Xia, Lijun Zhang, Xiaowei Chen, Yuqin Huang, Dalin Sun, Fang Fang, Zaiping Guo, and Xuebin Yu
}




\section{Carbon Hollow Nanobubbles on Porous Carbon}

\section{Nanofibers: An Ideal Host for High-Performance}

\section{Sodium-Sulfur Batteries and Hydrogen Storage}

Guanglin Xia,${ }^{a, b}$ Lijun Zhang, ${ }^{a}$ Xiaowei Chen, ${ }^{c}$ Yuqin Huang, ${ }^{a}$ Dalin Sun, ${ }^{a, d}$ Fang Fang, ${ }^{* a}$

Zaiping Guo, ${ }^{* a}$ and Xuebin Yu $u^{*, a, d}$

aDepartment of Materials Science, Fudan University, Shanghai 200433, China

${ }^{b}$ Institute for Superconducting and Electronic Materials, University of Wollongong, North

Wollongong, NSW 2522, Australia

${ }^{c}$ Department of Physics, School of Science, Jimei University, Xiamen 361021, China

${ }^{d}$ Shanghai Innovation Institute for Materials, Shanghai 200444, China

* To whom correspondence should be addressed.

E-mail: yuxuebin@fudan.edu.cn; zguo@uow.edu.au; f fang@,fudan.edu.cn 


\section{ABSTRACT}

One-dimensional (1D) carbon nanostructures have been intensively investigated because of their intriguing features and great potential for practical application in various fields. This paper reports the controllable fabrication of carbon hollow nanobubbles on porous carbon nanofibers (CHNBs@PCNFs) through a general electrospinning strategy, with metal azides serving as both a bubbling and a porogen reagent. The strong repulsive forces resulting from the intense release of $\mathrm{N}_{2}$ from the decomposition of metal azides upon carbonization leads to the uniform formation of porous carbon nanofibers (PCNFs), which could be facile tuned by heating rates and the amount of the bubbling reagent, simultaneously constructed with carbon hollow nanobubbles (CHNBs) on the surface. Density functional theory calculations reveal the strong interactions between terminal $\mathrm{Na}$ atoms in sodium polysulfides and $\mathrm{N}$ and $\mathrm{O}$ atoms doped into CHNBs@PCNFs, which could effectively alleviate the shuttle effect of Na-S batteries via adsorbing and trapping polysulfides. With strong adsorption capability of sodium polysulfides and high electrical conductivity, these CHNBs@PCNFs are demonstrated to be an ideal sulfur host in room-temperature sodium-sulfur batteries, which delivers a high reversible capacity of $256 \mathrm{~mA} \mathrm{~h} \mathrm{~g}^{-1}$ (specific energy density $384 \mathrm{~W} \mathrm{~h} \mathrm{~kg}^{-1}$ ) with a low decay rate of $0.044 \%$ per cycle

at 2 C-rate. When CHNBs@PCNFs used as functional supports for $\mathrm{MgH}_{2}$ nanoparticles, a significantly enhanced hydrogen storage performance was achieved. The present work represents a critically important step in advancing the electrospinning technique for generating $1 \mathrm{D}$ carbon nanostructures in a facile and universal manner.

Keywords: Porous carbon, Nanobubble, Hydrogen storage, Magnesium hydride, Nanofibers. 


\section{Introduction}

The ever increasing global demand for renewable energy generated by green but intermittent power sources, such as wind and solar, requires efficient energy storage systems. Owing to their high energy densities, electrochemical energy storage and hydrogen storage materials have received intense attentions. ${ }^{[1-3]}$ As an energy carrier, hydrogen can in principle be produced from a diverse range of renewable sources. ${ }^{[4]}$ More importantly, because it has the highest gravimetric energy storage density $\left(142 \mathrm{MJ} \mathrm{kg}^{-1}\right)$ and it is the most environmentally benign fuels with the production of only water from combustion, hydrogen has been widely investigated as a universal energy carrier that could enable the transition to renewable energy sources. ${ }^{[5]}$ The safe and efficient storage of hydrogen is one of the key challenges towards the development of sustainable hydrogen economy ${ }^{[6]}$

On the other hand, metal-sulfur batteries (e.g., Li-S and Na-S batteries) have also been recognized as a promising technology for stationary energy storage due to the high theoretical capacity $\left(1672 \mathrm{~mA} \mathrm{~h} \mathrm{~g}^{-1}\right)$ of sulfur cathode material with high natural abundance and low cost. ${ }^{[7-}$

9] The limited lithium resources, uneven distribution and hence relatively high price, however, limit the large-scale applications of Li-S batteries. ${ }^{[10]}$ In this regard, sodium has been considered as a low-cost alternative to lithium as anode, featuring comparable mechanism of chemical insertion and reactivity, due to both the low cost and the rich abundance of Na sources. ${ }^{[10]}$ Among them, the high-temperature molten-electrode sodium-sulfur (Na-S) battery has been regarded as one of the most successful energy storage technologies owing to its application in stationary energy storage systems for several decades. ${ }^{[9,11]}$ Unfortunately, a high operating temperature above $300{ }^{\circ} \mathrm{C}$ is required for melting the ceramic electrolyte, $\mathrm{NaAl}_{11} \mathrm{O}_{17}$, in 
traditional Na-S batteries, which leads to high cost and serious safety problems due to melting of the $\mathrm{Na}$ and $\mathrm{S}$ electrodes and limits their widespread applications. ${ }^{[12]}$ In order to avoid these disadvantages, rechargeable room-temperature $\mathrm{Na}-\mathrm{S}$ batteries are proposed with a liquid polymer as the electrolyte. ${ }^{[13,14]}$ The operation of a Na-S battery at ambient temperature, however, suffers from low reversible capacity and extremely fast capacity fading during cycling due to the intrinsic electronically insulating properties of sulfur and the fracturing of the electrodes caused by the large volumetric changes occurring between sulfur and sodium sulfide. ${ }^{[15]}$ Another obstacle obstructing the practical development of Na-S batteries relates to sodium polysulfides (NaPSs), the highly soluble intermediate products, which lead to rapid sulfur loss, the unfavorable shuttle effect, and also the subsequent accumulation of electrically inert reduction products on the electrodes. ${ }^{[16-19]}$

In order to address these problems, porous carbon materials, including carbon hollow nanospheres, carbon nanotubes, and carbon nanofibers, have been proposed and investigated as supporting materials for sulfur cathodes to improve the electrochemical performance of $\mathrm{Na}-\mathrm{S}$ batteries. $^{[15,20-24]}$ A carbon matrix could significantly improve the electrical conductivity of sulfur cathodes, leading to the enhanced utilization of the active sulfur materials. ${ }^{[7,8,25]}$ In addition, the flexible nature and the high pore volume of carbon is beneficial for accommodating the large volume changes of sulfur associated with sodium insertion and extraction. ${ }^{[20]}$ Recently, increasing attention has been paid to the synthesis of one-dimensional (1D) carbon nanofibers (CNFs) with designed porous architecture for supporting electrochemically active materials, which is mainly attributable to its robust geometry to maintain their functionalities during chemical reactions and provide efficient paths for electron and mass transport. ${ }^{[6-28]}$ Significantly improved specific capacities, however, are usually only obtained when a high percentage of 
carbon matrix is introduced because of the limited pore volume of current CNFs. ${ }^{[29,30]}$ This leads to low sulfur loading in the cathode materials and hence low energy density, which makes them less attractive for practical applications. Additionally, due to the weak interaction between nonpolar CNFs and polar NaPSs, rapid capacity decay is still present during discharge and charge cycling, which is mainly due to the dissolution of the reaction intermediate $\mathrm{NaPSs},{ }^{[23]}$ so that the cycling stability of $\mathrm{Na}-\mathrm{S}$ batteries remains a great challenge for practical applications. Therefore, it is necessary to develop new porous CNFs (PCNFs) that possess both high volumes to effectively accommodate the volume changes of sulfur in high-mass-loading Na-S batteries and effective chemical interaction with the NaPSs to alleviate the shuttle effect, which is crucial to the design of advanced Na-S batteries with high loading and utilization of sulfur, long cycling life, and good rate capability.

Bearing these considerations in mind, here, we have designed and successfully synthesized PCNFs with a hierarchical architecture, i.e., controllable carbon hollow nanobubbles constructed on porous carbon nanofibers, through bubbling-assisted electrospinning followed by a simple stepwise calcination process. The as-electrospun nanofibers (NFs) were first obtained by electrospinning a homogeneous solution of poly(vinyl alcohol) (PVA) containing the bubbling and porogen reagent $\mathrm{LiN}_{3}$ (Figure 1). By simply tuning the amount of $\mathrm{LiN}_{3}$ and the heating rate during carbonization, PCNFs, containing micropores, mesopores, and macropores, were controllably fabricated, with the simultaneous formation of carbon hollow nanobubbles (CHNBs) with a diameter of 10-60 nm on their surfaces, which provide large void space for sulfur accommodation. Moreover, the integrated 1D PCNFs, which constitute continuous electron conductive pathways, could effectively facilitate rapid electron and ion transport within insulating $\mathrm{S}$ nanoparticles accommodated by isolated CHNBs. Furthermore, density functional 
theory (DFT) calculations verify the strong chemical adsorption capability of CHNBs@PCNFs for NaPSs, induced by the strong interactions between terminal $\mathrm{Na}$ atoms in sodium polysulfides and $\mathrm{N}$ and $\mathrm{O}$ atoms doped in during the carbonization process, which effectively alleviate the shuttle effect of NaPSs. Thus, when employing CHNBs@PCNFs as the sulfur host for Na-S batteries, the S(CHNBs@PCNFs) cathodes exhibit a significantly improved utilization rate of sulfur, and good cycling stability and rate capability. Additionally, the robust porous structure and the catalytic effects of CHNBs@PCNFs are successfully exploited as a functional structural framework to support $\mathrm{MgH}_{2}$ nanoparticles, which exhibit complete hydrogenation at a temperature as low as $250{ }^{\circ} \mathrm{C}$ within only $15 \mathrm{~min}$.

\section{Results and discussion:}

To construct $\mathrm{CHNBs}$ on PCNFs, PVA nanofibers containing the bubbling agent (i.e., $\mathrm{LiN}_{3}$ ) were first fabricated via a scalable and simple electrospinning technique. Scanning electron microscope (SEM) images of the as-electrospun PVA-LiN 3 NFs at different magnifications in Figure 2a reveal that the as-obtained PVA-LiN 3 NFs are long and continuous, with a smooth surface and a relatively uniform diameter of about $300 \mathrm{~nm}$, which are analogous to the PVA NFs without the addition of $\mathrm{LiN}_{3}$ (Figure $\mathrm{S} 1$ in the Supporting Information). After the stepwise carbonization, the as-formed CNFs from PVA NFs without $\mathrm{LiN}_{3}$ have smooth surfaces with an average diameter of $\sim 180 \mathrm{~nm}$ (Figure S2a). By comparison, although the calcination of PVA NFs with the addition of $\mathrm{LiN}_{3}$ through the same procedure leads to the fabrication of CNFs with the same basic fibrous structure, a plethora of nanobubbles with various diameters ranging from 10 to $60 \mathrm{~nm}$ are uniformly and densely distributed on the surfaces of the as-prepared CNFs, which have a diameter of $\sim 200 \mathrm{~nm}$ (Figures $2 \mathrm{~b}$ and c). Transmission electron microscope (TEM) images (Figures $2 \mathrm{~d}$ and e) further confirm that these carbon nanobubbles are hollow and that the 
shells are very thin, with a thickness of only $\sim 2 \mathrm{~nm}$. Furthermore, the presence of pores with diameters of around 10-30 nm could be observed inside the body of the CNFs (Figure 2d), which could be attributed to the vigorous decomposition of $\mathrm{LiN}_{3}$, approaching the center of the PVA NFs, while no porous structure was observed for CNFs obtained from the decomposition of the as-electrospun PVA nanofibers without the presence of $\mathrm{LiN}_{3}$ (Figure S2b). The presence of long and continuous PCNFs during TEM observations after the vigorous scratching and ultrasonic treatment used for preparing the samples indicates its high structural robustness. Selected area electron diffraction (SAED) patterns (inset of Figure 2d) show dispersing diffraction rings, demonstrating the amorphous nature of CHNBs@PCNFs after washing and drying, which coincides well with XRD results (Figure S3). The porous texture of CHNBs@PCNFs was further investigated by nitrogen adsorption/desorption isotherms at $77 \mathrm{~K}$ (Figure S4a), which reveal a specific surface area of $420 \mathrm{~m}^{2} \mathrm{~g}^{-1}$ and a large pore volume of $1.64 \mathrm{~cm}^{3} \mathrm{~g}^{-1}$, respectively. The distribution of pore sizes (Figure $\mathrm{S} 4 \mathrm{~b}$ ) determined from the adsorption branches is wide, with many peaks in the $2-100 \mathrm{~nm}$ range. Three regions can be identified: a) micropores $(0-2 \mathrm{~nm}), \mathrm{b})$ mesopores $(8-25 \mathrm{~nm})$, and c) macropores $(50-68 \mathrm{~nm})$, which confirms the hierarchical porous characteristics of the CHNBs@PCNF texture and agrees well with the SEM/TEM observations. In strong constrast, without the presence of $\mathrm{LiN}_{3}$, the specific surface area of the as-synthesized CNFs is only around $208 \mathrm{~m}^{2} \mathrm{~g}^{-1}$ with an average pore size of $\sim 2 \mathrm{~nm}$ (Figure S5).

In order to gain insight into the development of this unique structure, the thermal decomposition of as-electrospun PVA NFs with and without the bubbling agent was measured by thermogravimetric analysis (TGA) in $\mathrm{N}_{2}$ atmosphere (Figure $\mathrm{S} 6$ ). No violent weight loss was observed for them below $170{ }^{\circ} \mathrm{C}$, indicating that both samples were thermally stable under these conditions. Subsequently, a strong weight loss was detected in the range of $170-240{ }^{\circ} \mathrm{C}$ for the 
PVA-LiN $\mathrm{NFs}_{3}$, which could be attributed to the strong $\mathrm{N}_{2}$ evolution resulting from the explosive decomposition of the bubbling agent ${ }^{[31]}$, while no decomposition behavior was observed for the PVA NFs without $\mathrm{LiN}_{3}$. Further weight loss between $240{ }^{\circ} \mathrm{C}$ and $650{ }^{\circ} \mathrm{C}$ was induced by the carbonization of PVA NFs to form carbon nanofibers. Therefore, in order to obtain structurally integrated three-dimensional (3D) PCNFs, a stepwise annealing strategy was applied to calcine the as-electrospun PVA-LiN 3 NFs by tuning the decomposition rate of $\mathrm{LiN}_{3}$ according to the TGA results. First, the as-electrospun PVA-LiN ${ }_{3}$ films were stabilized and dewatered under $\mathrm{N}_{2}$ atmosphere at $150{ }^{\circ} \mathrm{C}$ for $1 \mathrm{~h}$, and the 1D fibrous morphology was well maintained (Figure S7). Subsequently, the dried PVA-LiN 3 NFs were slowly heated to $240{ }^{\circ} \mathrm{C}$ with a heating rate of $0.5{ }^{\circ} \mathrm{C} \mathrm{min}-1$ and kept at $240{ }^{\circ} \mathrm{C}$ for $2 \mathrm{~h}$ in $\mathrm{N}_{2}$ atmosphere to ensure the complete decomposition of $\mathrm{LiN}_{3}$. The color of the as-electrospun films accordingly changes from yellow to ruby red, which is the characteristic color of the $\mathrm{Li}_{3} \mathrm{~N}$ resulting from the decomposition of metal azides, and the SEM images verify the formation of massive nanobubbles on the surfaces of the 1D fibrous structures at this stage (Figure S8). XRD patterns (Figure S3) also confirm the formation of $\mathrm{Li}_{3} \mathrm{~N}$ upon thermal heating to $240{ }^{\circ} \mathrm{C}$. These results directly demonstrate that the generation of nanobubbles is derived from the repulsive force of the $\mathrm{N}_{2}$ that is liberated by the decomposition of $\mathrm{LiN}_{3}$. Finally, after carbonization at $650{ }^{\circ} \mathrm{C}$ for $180 \mathrm{~min}$, the color of the films becomes black, and the 1D architecture is well maintained, in which CHNBs are uniformly formed on the surfaces of PCNFs, leading to the formation of CHNBs@PCNFs.

To further unravel the role of $\mathrm{LiN}_{3}$ in tuning the nanostructure of the as-synthesized PCNFs, a series of control experiments were carried out. It could be clearly observed that, as expected, the size and density of CHNBs increased, in general, with an increase in the weight ratio of $\mathrm{LiN}_{3}$ to PVA in the electrospinning precursors (Table S1). More interestingly, based on the fact that the 
generation of CHNBs and hierarchical pores inside the CNFs results from the decomposition of $\mathrm{LiN}_{3}$, it is proposed that the morphology of CHNBs could be easily controlled by tuning the decomposition rate of $\mathrm{LiN}_{3}$, which was facilely adjusted by changing the heating rate during the process of carbonization. SEM images (Table S2) demonstrate that, when the weight ratio of $\mathrm{LiN}_{3}$ to PVA is fixed at $21.5 \%$, the increased diameter and density of the as-formed CHNBs are closely correlated with decreased heating rates within a certain range from 1 to $0.5{ }^{\circ} \mathrm{C} \mathrm{min}^{-1}$. Many open pores were generated on the surfaces of the CNFs instead of the formation of CHNBs due to the explosive release of nitrogen when the heating rate is $2{ }^{\circ} \mathrm{C} \mathrm{min}^{-1}$. This result obviously confirms that the heating rate plays a critically important role in tuning the formation of CHNBs on the surfaces of PCNFs, i.e., the explosive release of $\mathrm{N}_{2}$ with a relatively higher heating rate will push away the PVA surrounding $\mathrm{LiN}_{3}$ and then burst out, leading to the formation of pores on the surface, while the gentle desorption of $\mathrm{N}_{2}$ at a lower heating rate could slowly blow up the surrounding PVA, leading to the formation of CHNBs on the surface after carbonization. Therefore, the nanoarchitecture of the as-synthesized CHNBs@PCNFs could be facilely controlled by simply changing the concentration of $\mathrm{LiN}_{3}$ and the heating rate for carbonization, which provides great potential for their applications in various fields.

To expand the application of $\mathrm{LiN}_{3}$ as the pore generator, polyvinyl pyrrolidone (PVP) was further adopted as the carbon precursor with the consideration of the solubility of PVP in water with $\mathrm{LiN}_{3}$ and the wide application of PVP in electrospinning. Although the calcination of PVP$\mathrm{LiN}_{3}$ nanofibers through the same procedure leads to the fabrication of CNFs with the fibrous structure, no pores as observed in the CHNBs@PCNFs are present (Figure S9). TGA results exhibit that, in contrast with PVA-LiN 3 NFs, there is no violent decomposition step of $\mathrm{LiN}_{3}$ during the carbonization of PVP-LiN 3 NFs (Figure S6). Moreover, it could be clearly observed 
that the weight loss of PVP-LiN 3 NFs is much lower than that of pure PVP NFs. These results demonstrate the reaction between $\mathrm{LiN}_{3}$ and PVP upon thermal heating, which leads to the loss of $\mathrm{LiN}_{3}$ as the pore generator.

The carbon structure of CHNBs@PCNFs was subsequently investigated by Raman spectroscopy, in which two broad peaks located at 1326 and $1584 \mathrm{~cm}^{-1}$ could be assigned to typical D- and G-bands of carbon, respectively (Figure S10). The intensity ratio of the D to the G-band of CHNBs@PCNFs was calculated to be 1.08, indicating the presence of abundant defects and vacancies, which could not only promote the diffusion of sodium ions, but also provide more intercalation sites for sodium storage. ${ }^{[32]}$ The chemical composition and chemical bonding states of CHNBs@PCNFs were investigated by X-ray photoelectron spectroscopy (XPS) and elemental analysis. XPS spectra of the CHNBs@PCNFs composite exhibit the presence of C, $\mathrm{N}$, and $\mathrm{O}$ (Figure S11), with no other impurities detected. N doping with a dopant content of 5.2\% is derived from the reaction between the $\mathrm{Li}_{3} \mathrm{~N}$ generated by the decomposition of $\mathrm{LiN}_{3}$ and PVA during the carbonization process. ${ }^{[33]}$ It could be supported by XRD results, which confirms the disappearance of $\mathrm{Li}_{3} \mathrm{~N}$ and the formation of $\mathrm{Li}_{2} \mathrm{CO}_{3}$ before washing and drying (Figure $\mathrm{S} 3$ ). The C 1s XPS profile of CHNBs@PCNFs demonstrates the presence of $\mathrm{C}-\mathrm{C} / \mathrm{C}=\mathrm{C}$ bonds and oxygencontaining functional groups, as well as the formation of $\mathrm{C}-\mathrm{N}$ bonds (Figure S12a), while the high-resolution N 1s spectrum of CHNBs@PCNFs could be resolved into three different peaks, which correspond to pyridinic $\mathrm{N}$ at $399.2 \mathrm{eV}$, pyrrolic $\mathrm{N}$ at $400.5 \mathrm{eV}$, and graphitic $\mathrm{N}$ at 401.9 $\mathrm{eV}$, respectively (Figure $\mathrm{S} 12 \mathrm{~b}$ ). The doping of these electron-rich heteroatoms, i.e., $\mathrm{O}$ and $\mathrm{N}$, could produce abundant active sites and tune the surface electronic structure of the carbon materials, thus promoting the surface affinity for polysulfides. ${ }^{[34-36]}$ The ability of CHNBs@PCNFs to absorb NaPSs was first evaluated by ultraviolet-visible (UV-vis) 
spectroscopy before and after exposure to CNFs and CHNBs@PCNFs (Figure 3a). The solution exposed to CHNBs@PCNFs exhibited much lower peak intensities of characteristic bands of NaPSs than those containing CNFs or the fresh solution without additives, indicating the strong adsorption capability of CHNBs@PCNFs derived from nitrogen- and oxygen-doping. Further evidence is provided by the lighter color of the NaPSs solution after exposure to CHNBs@PCNFs (inset of Figure 3a), which becomes almost colorless within 30 min. By comparison, the solution exposed to CNFs remains intensely yellow, suggesting extremely weak interaction between the nonpolar CNFs and the polar NaPSs.

In order to examine the remarkable adsorption of heteroatom-doped CHNBs@PCNFs on NaPSs, first principles calculations were conducted based on density functional theory (DFT) to obtain the binding geometries and energies. As shown in Figure $3 \mathrm{~b}$, the binding energies of $\mathrm{Na}_{2} \mathrm{~S}$, $\mathrm{Na}_{2} \mathrm{~S}_{4}$, and $\mathrm{Na}_{2} \mathrm{~S}_{6}$ systems over the $\mathrm{N}$, O-codoped carbon were calculated to be $3.2,1.18$, and $1.16 \mathrm{eV}$, respectively, which are much higher than the corresponding values on $\mathrm{N}$-doped, Odoped, and non-doped carbon (Figure S13). This indicates that nitrogen and oxygen codoping into carbon surfaces is more effective for adsorbing and trapping various polysulfides than the other carbon-based surfaces, which could effectively alleviate the shuttle effect of Na-S batteries. The optimized geometry indicates that the $\mathrm{Na}-\mathrm{O}$ distance in $\mathrm{Na}_{2} \mathrm{~S}_{4}-\mathrm{N}$, O-codoped carbon is 2.428 $\AA$, quite close to that in the crystalline phase of $\mathrm{Na}_{2} \mathrm{O}(2.403 \AA)$, which suggests the formation of the Na-O ionic bond, while the Na-N distance is $3.018 \AA$, only $0.649 \AA$ longer than that in the crystalline phase of $\mathrm{Na}_{3} \mathrm{~N}$ (Figure S14). More interestingly, the Na-N and $\mathrm{Na}-\mathrm{O}$ distances in the adsorption geometry of $\mathrm{Na}_{2} \mathrm{~S}_{6}$ on $\mathrm{N}, \mathrm{O}$-codoped carbon are 2.387 and $2.414 \AA$, respectively, and both are comparable to the corresponding bond lengths in the crystalline phases of $\mathrm{Na}_{3} \mathrm{~N}$ and $\mathrm{Na}_{2} \mathrm{O}$, validating the formation of $\mathrm{Na}-\mathrm{N}$ and $\mathrm{Na}-\mathrm{O}$ ionic bonds in this system. The charge density 
distribution maps (Figures 3c, d) demonstrate that terminal $\mathrm{Na}$ atoms in NaPSs tend to directly bind to $\mathrm{N}$ and $\mathrm{O}$ atoms that have been dual-doped into carbon with a distinctive electron concentration between them, which suggests that the lone pair electrons of $\mathrm{N}$ and $\mathrm{O}$ atoms could interact with $\mathrm{Na}$ toms. This leads to a strong Na-N/O ionic bond interaction between he soluble NaPSs and the N, O-codoped carbon, which could effectively preserve the active materials and restrict the shuttle effect.

Considering its structural stability, with sufficient pore volume for accommodating a high loading ratio of sulfur to improve the energy density of Na-S batteries, CHNBs@PCNFs synthesized according to the optimized $\mathrm{LiN}_{3} / \mathrm{PVA}$ ratio of $21.5 \%$ under a heating rate of $0.5{ }^{\circ} \mathrm{C}$ $\min ^{-1}$ were selected for further investigation. Sulfur was infiltrated into the CHNBs@PCNFs via a facile melt-infiltration method, and it could be clearly observed that the as-prepared composite maintains a long, continuous fibrous structure (Figure 3e). In addition, the diameter and shell thickness of CHNBs were well preserved, and no aggregated sulfur particles were exposed on the surface, indicating the complete diffusion of sulfur into the void spaces of CHNBs@PCNFs. The darker region inside every CHNB after sulfur infiltration compared to the pure CHNBs indicates the successful loading of sulfur (Figure 3f). Energy dispersive spectroscopy (EDS) elemental mapping obviously verifies the uniform distribution of $\mathrm{C}, \mathrm{N}$, and $\mathrm{S}$ in the CHNBs@PCNFs (Figure 3g), and the signal of sulfur is also uniformly well matched with the CHNBs on PCNFs. These results demonstrate the homogeneous confinement of sulfur within the entire CHNBs@PCNFs sample. Further evidence is provided by the drastic decrease in surface area and pore volume after sulfur infiltration according to the nitrogen absorption-desorption isotherms (Figure S4). It is interesting to notice the partial preservation of the pores and large empty volume after the infiltration process, which agrees well with the TEM results, and could 
effectively accommodate the volume changes of $\mathrm{S}$ in the electrochemical reactions during cycling. The X-ray diffraction (XRD) pattern of S/(CHNBs@PCNFs) composite reveals the presence of weak signals belonging to sulfur with the same orthorhombic structure as elemental sulfur powder (Figure S15). The sulfur content was determined to be approximately $71.2 \%$ by thermogravimetric analysis (TGA) (Figure S16). It should be noted that S/(CHNBs@PCNFs) exhibits a higher sulfur vaporization temperature than pure sulfur or $\mathrm{S} / \mathrm{CNFs}$, which suggests the strong absorption of sulfur by the CHNBs@PCNFs and the incorporation of sulfur into the small pores of the carbon framework. Additionally, the signal of sulfur is undetectable in Raman spectra of S/(CHNBs@PCNFs) (Figure S10), suggesting a good distribution of S inside CHNBs@PCNFs without any obvious aggregation, corresponding well with the SEM and TEM results.

The electrochemical properties of $\mathrm{S} /(\mathrm{CHNBs} @ \mathrm{PCNFs})$ cathodes were evaluated by assembling them into Na-S batteries with Na-foil as an anode. Cyclic voltammetry (CV) of the resultant Na-S battery was conducted in the voltage range from 1.2-2.8 $\mathrm{V}$ at a scan rate of 0.1 $\mathrm{mV} \mathrm{s}^{-1}$. In the first cycle, the cathodic peaks at 2.22 and $1.42 \mathrm{~V}$ are attributed to the reduction of sulfur to long-chain NaPSs (e.g., $\mathrm{Na}_{2} \mathrm{~S}_{n}, 4 \leq n \leq 8$ ) and short-chain sodium sulfides (e.g., $\mathrm{Na}_{2} \mathrm{~S}_{2}$ ) or sodium sulfide $\left(\mathrm{Na}_{2} \mathrm{~S}\right) .{ }^{[17,37]}$ XRD patterns (Figure $\left.\mathrm{S} 17\right)$ demonstrate the formation of NaPSs and $\mathrm{Na}_{2} \mathrm{~S}$ in the discharged products of $\mathrm{S} /(\mathrm{CHNBs} @ \mathrm{PCNFs})$ cathodes after being discharged to $2.0 \mathrm{~V}$ and $1.2 \mathrm{~V}$, respectively. In addition, as shown in the HRTEM images (Figure S18), after being discharged to $2.0 \mathrm{~V}$, the d-spacings of the crystalline nanoparticles in the S/(CHNBs@PCNFs) cathodes were measured to be 0.44 and $0.48 \mathrm{~nm}$, corresponding to the (100) planes of $\mathrm{Na}_{2} \mathrm{~S}_{5}$ and the (200) planes of $\mathrm{Na}_{2} \mathrm{~S}_{4}$, while the d-spacings of the $\mathrm{S} /(\mathrm{CHNBs} @ \mathrm{PCNFs})$ cathodes being dicharged to $1.2 \mathrm{~V}$ could be acribed to the (111) planes of $\mathrm{Na}_{2} \mathrm{~S}$, in good 
agreement with the XRD results. High-resolution S $2 p$ spectra provides further evidence to the formation of $\mathrm{Na}_{2} \mathrm{~S}$ as well as the absence of sulfur after the initial full discharge process (Figure S19). In the anodic process, two peaks at around 1.87 and $2.41 \mathrm{~V}$, corresponding to the conversion of sodium sulfides to polysulfides and sulfur, respectively, are observed. ${ }^{[17,37]}$ After the initial full charge process, although no visible peak is observed in the XRD patterns of S/(CHNBs@PCNFs) cathodes, which indicates the amorphous state of the charged products without long-range order, the characteristic peaks belonging to sulfur could be clearly observed in the XPS spectra with the disappearance of NaPSs and $\mathrm{Na}_{2} \mathrm{~S}$, which suggests the transformation of NaPSs and $\mathrm{Na}_{2} \mathrm{~S}$ back to sulfur. Those results confirm the conversion reaction of sulfur upon cyling charge-discharge process, accounting for the reversible electrochemical capacity. After the electrochemical activation during the first cycle, two pairs of obvious redox peaks at 2.23/2.41 and 1.52/1.87 V , corresponding to reversible transitions between elemental sulfur, long-chain NaPSs, and short chain sodium sulfide, respectively, are present in the subsequent sweeps. The CV profiles remained unchanged after the first two cycles without any noticeable peak shift, indicating the excellent stability and high reversibility of S/(CHNBs@PCNFs). The galvanostatic charge/discharge behavior of the S/(CHNBs@PCNFs) cathode was subsequently investigated at a rate of $0.1 \mathrm{C}\left(1 \mathrm{C}=1675 \mathrm{~mA} \mathrm{~g}^{-1}\right)$. Two plateaus were observed at $\sim 1.48$ and $2.22 \mathrm{~V}$ in the discharge process, which were attributed to the two-stage reduction of elemental sulfur to long-chain NaPSs and then to $\mathrm{Na}_{2} \mathrm{~S}_{2} / \mathrm{Na}_{2} \mathrm{~S}$, respectively, which agrees well with the CV results. The S/(CHNBs@PCNFs) cathode delivers an initial specific discharge capacity of $1214 \mathrm{~mA} \mathrm{~h} \mathrm{~g}^{-1}$ at the rate of $0.1 \mathrm{C}$, corresponding to $72.6 \%$ sulfur utilization, based on the theoretical value of sulfur $\left(1672 \mathrm{~mA} \mathrm{~h} \mathrm{~g}^{-1}\right)$, and gains a reversible specific capacity of $913 \mathrm{~mA} \mathrm{~h} \mathrm{~g}^{-1}$ in the first charge. It should be noted that the contribution of 
CHNBs@PCNFs could be ignored due to its low specific capacity of $48 \mathrm{~mA} \mathrm{~h} \mathrm{~g}^{-1}$ in the given potential range and its low weight ratio in the composite (Figure S20). These initial specific capacities are much higher than those of the S/CNFs (394 mA h g${ }^{-1}$ ) and sulfur (48 $\mathrm{mA} \mathrm{h} \mathrm{g}^{-1}$ ) electrodes (Figure 4c). The coulombic efficiency of the electrode quickly stabilizes to $>95 \%$ within 10 cycles. After 50 cycles, the S/(CHNBs@PCNFs) cathode still maintains a high reversible capacity of $786 \mathrm{~mA} \mathrm{~h} \mathrm{~g}^{-1}$, corresponding to a capacity retention of $65 \%$, which indicates its good cycling stability, which is due to the strong adsorption capability of CHNBs@PCNFs towards NaPSs. By comparison, the S/CNFs electrode exhibits much lower sulfur utilization and poor cycling performance.

To understand the role of CHNBs@PCNFs in improving the performance of Na-S batteries, the batteries were disassembled after 50 cycles of discharge and charge process and postmortem SEM analysis was conducted on the pure sulfur, S/CNFs and S/(CHNBs@PCNFs) electrodes (Figure S21). It is revealed that, due to the redeposition of insoluble $\mathrm{Na}_{2} \mathrm{~S} / \mathrm{Na}_{2} \mathrm{~S}_{2}$ species on the cathode surface, a thick layer of glassy discharge products is formed on the pure sulfur electrode with the serious aggregation of sulfur after cycling discharge and charge process derived from the redisposition of insoluble $\mathrm{Na}_{2} \mathrm{~S} / \mathrm{Na}_{2} \mathrm{~S}_{2}$ species, which is similar with the phenomenon observed in Li-S batteries. ${ }^{[38-40]}$ Although this phenomenon is alleviated to a certain extent when using CNFs as the host of sulfur, the aggregation of sulfur is still apparently present, which suggests the dissolution loss of active sulfur into the electrolyte due to the lack of spatial confinement and weak binding between CNFs and NaPSs during electrochemical reactions, leading to the inhomogeneous deposition of $\mathrm{Na}_{2} \mathrm{~S} / \mathrm{Na}_{2} \mathrm{~S}_{2}$ species. By comparison, no glassy discharge products on the surface of the $\mathrm{S} /(\mathrm{CHNBs} @ \mathrm{PCNFs})$ electrode after 50 cycles were observed and strong sulfur signals are uniformly distributed within the whole electrode, which 
indicates the effective absorption and trapping of polysulfides within CHNBs@PCNFs. Thus, the utilization of the active sulfur materials was significantly enhanced via adopting CHNBs@PCNFs as the host of sulfur. These results validate that CHNBs@PCNFs could effectively restrict the diffusion of soluble sodium polysulfides, which would improve the utilization of sulfur and the cycling stability.

Rate tests of S/(CHNBs@PCNFs) cathode at different current rates exhibited an excellent high-rate capability. On increasing the current rate to $0.2,0.5$, and $1 \mathrm{C}$, the $\mathrm{S} /(\mathrm{CHNBs} @ \mathrm{PCNFs})$ cathode could deliver high discharge capacity of 709, 598, and $487 \mathrm{~mA} \mathrm{~h} \mathrm{~g}^{-1}$, respectively. Even at a high current density of $2 \mathrm{C}$, a high reversible capacity of $304 \mathrm{~mA} \mathrm{~h} \mathrm{~g}^{-1}$ could still be achieved. After completing the $2 \mathrm{C}$ rate measurement, a high reversible capacity of $790 \mathrm{~mA} \mathrm{~h} \mathrm{~g}^{-1}$ was retained, indicating fast electrochemical reactions kinetics of the electrode and the good electrode integrity. In comparison, the average specific capacity of S/CNFs cathode fades drastically from 415 to $74 \mathrm{~mA} \mathrm{~h} \mathrm{~g}^{-1}$ as the rate increases from 0.1 to $2 \mathrm{C}$. The long-term cycling stability of the S/(CHNBs@PCNFs) cathode was also investigated at the high rate of 2 C, and it presented a high specific capacity as well as good cycling retention. After 400 cycles, the S/(CHNBs@PCNFs) cathode still retained a high capacity of $256 \mathrm{~mA} \mathrm{~h} \mathrm{~g}^{-1}$ (specific energy density: $384 \mathrm{~W} \mathrm{~h} \mathrm{~kg}^{-1}$ ) with a low decay rate of $0.044 \%$ per cycle. More importantly, the average Coulombic efficiency of the Na-S battery containing the S/(CHNBs@PCNFs) cathode was approaching $100 \%$ throughout the whole long-term cycling test, exhibiting great potential for the practical application of high-energy and long-life Na-S batteries. To the best of our knowledge, the rate and cycling performance of $\mathrm{S} /(\mathrm{CHNBs} @ \mathrm{PCNFs})$ is the best performance among carbonbased sulfur electrodes (Table S3). The excellent reversibility, high capacity, and superior cycling performance of $\mathrm{S} /(\mathrm{CHNBs} @ \mathrm{PCNFs})$ could be attributed to the unique features of 
CHNBs@PCNFs as sulfur host: (1) CHNBs on 1D PCNFs, with high aspect ratio and abundant pores, can effectively facilitate the transportation of electrolyte and buffer the mechanical stresses generated by the volume variation of S cathode during cycling; (2) CHNBs could not only provide abundant room to accommodate sulfur, but also act as a polysulfide reservoir through chemical adsorption to alleviate the shuttle effect, leading to significantly improved cycling stability; (3) The integrated 1D structure of PCNFs provides continuous conductive pathways, which could aid in rapid electron transfer within the insulating sulfur confined in the isolated bubbles, i.e., CHNBs, resulting in high sulfur utilization and hence reversible capacity.

The unique architecture of CHNBs@PCNFs, i.e., large pore volume, hierarchical pores, and robust structure, could be considered as containing promising nanoscale building blocks for functional materials. To elucidate, in this work, CHNBs@PCNFs were further investigated as a stable structural framework to support $\mathrm{MgH}_{2}$ nanocrystals (NCs) as hydrogen storage materials. Due to its high gravimetric $\left(7.6 \mathrm{wt} . \% \mathrm{H}_{2}\right)$ and volumetric $\left(109 \mathrm{~g} \mathrm{H}_{2} \mathrm{~L}^{-1}\right)$ hydrogen capacities, high abundance, low cost, and high safety, $\mathrm{MgH}_{2}$ represents a highly promising candidate for hydrogen storage. ${ }^{[3]}$ The practical application of $\mathrm{MgH}_{2}$, however, is strongly obstructed by its thermodynamic stability and slow kinetics for reversible hydrogen storage. ${ }^{[41]}$ One of the most effective strategies to relieve the kinetic barrier and/or thermodynamic stability is to disperse $\mathrm{MgH}_{2}$ nanocrystals (NCs) into porous hosts, which could directly decrease the solid-state diffusion distance for hydrogen storage and increase the surface-to-volume ratio of particles, leading to a significantly enhanced hydrogen storage performance. ${ }^{[42-44]}$ Nonetheless, owing to the lack of control over the infiltration process and the highly limited porosity of the adopted hosts, inhomogeneous particle sizes, irregular spatial distributions, and low infiltration efficiency hinder the application of this strategy for improving the hydrogen storage properties of $\mathrm{MgH}_{2}{ }^{[45]}$ 
The unique structure and nature of CHNBs@PCNFs, however, is capable of dispersing $\mathrm{MgH}_{2}$ NCs and further improving their hydrogen storage performance. First, the hierarchical porous structure of CHNBs@PCNFs with large pore volume provides sufficient void space for anchoring $\mathrm{MgH}_{2} \mathrm{NCs}$. More importantly, the favorable interaction between the precursor of $\mathrm{MgH}_{2}$, i.e., di-n-butylmagnesium, and the carbon framework could ensure the homogeneous distribution of the thus-formed $\mathrm{MgH}_{2}$ NCs from di-n-butylmagnesium on CHNBs@PCNFs and promote further reduction of particle size. ${ }^{[46]}$ Furthermore, the catalytic effect of carbon in CHNBs@PCNFs could play an important role in further enhancing the hydriding and dehydriding performance of $\mathrm{MgH}_{2}{ }^{[47,48]}$ and the presence of abundant open channels in CHNBs@PCNFs would facilitate fast transportation of hydrogen and the adopted precursors.

As shown in Figure 5a, $\mathrm{MgH}_{2}$ NCs supported on CHNBs@PCNFs were realized by the selfassembly strategy using solvothermal treatment of di-n-butylmagnesium solution in cyclohexane under 3.5 MPa hydrogen pressure. ${ }^{[46]}$ After solvothermal treatment in cyclohexane at $200{ }^{\circ} \mathrm{C}$ for $24 \mathrm{~h}, \mathrm{CHNBs} @$ PCNFs retain their primary morphology owing to its robust structure, and the surface of CHNBs is no longer smooth but has become an anchoring site for a multitude of nanoparticles (Figure 5b). From the high-magnified TEM image (Figure 5c), the average particle size of $\mathrm{MgH}_{2} \mathrm{NCs}$ is measured to be approximately $5.1 \mathrm{~nm}$ (Figure S22), which could significantly improve the hydrogen storage properties of $\mathrm{MgH}_{2}$, owing to shortened diffusion distances and increased surface energy ${ }^{[49-51]}$, while a certain amount of space between these $\mathrm{MgH}_{2}$ NCs that are homogenously anchored on the CHNBs of CHNBs@PCNFs $\left(\mathrm{MgH}_{2} /(\mathrm{CHNBs} @ \mathrm{PCNFs})\right)$ could effectively alleviate the agglomeration and growth of the $\mathrm{MgH}_{2} \mathrm{NCs}$ during thermal heating for hydrogen storage. The high-resolution TEM (HRTEM) image (inset of Figure 5c) confirms that the lattice fringes of the nanoparticles have a spacing of 
$\sim 0.250 \mathrm{~nm}$, which corresponds well with the (101) planes of $\mathrm{MgH}_{2}$ and is consistent with the XRD (Figure S23) patterns. The elemental mapping in Figure 5d confirms that the $\mathrm{C}$ and $\mathrm{Mg}$ maps correspond well with the structure of CHNBs@PCNFs, demonstrating the homogeneous distribution of $\mathrm{MgH}_{2} \mathrm{NCs}$ both on the CHNBs and inside the PCNFs.

The dehydriding process for $\mathrm{MgH}_{2} / \mathrm{CHNBs@PCNFs}$ was first characterized by TGA and mass spectroscopy (MS). It should be noted that all the hydrogen capacity is calculated on the basis of $\mathrm{MgH}_{2}$ in the composite (Figure 6a). The ball-milled $\mathrm{MgH}_{2}$ and CHNBs@PCNFs exhibits a dehydrogenation peak at $323{ }^{\circ} \mathrm{C}$ (Figure $6 \mathrm{~b}$ ), $48{ }^{\circ} \mathrm{C}$ lower than for bulk $\mathrm{MgH}_{2}$, which demonstrates the catalytic role of CHNBs@PCNFs on the hydrogen storage process of $\mathrm{MgH}_{2}$. In strong contrast, $\mathrm{H}_{2}$ evolution of $\mathrm{MgH}_{2} /(\mathrm{CHNBs} @ \mathrm{PCNFs})$ occurs in the temperature range of 205-320 ${ }^{\circ} \mathrm{C}$ with a peak temperature of $265{ }^{\circ} \mathrm{C}$ (Figure 6b), $23{ }^{\circ} \mathrm{C}$ and $61{ }^{\circ} \mathrm{C}$ lower than for $\mathrm{MgH}_{2} \mathrm{NCs}$ without CHNBs@PCNFs and the ball-milled composite, respectively. This verifies the significant synergistic role of both the catalytic effects of CHNBs@PCNFs and the reduction of particle size down to the nanometer range towards improving the dehydrogenation process of $\mathrm{MgH}_{2}$. The dehydriding and hydriding kinetics of $\mathrm{MgH}_{2} /(\mathrm{CHNBs} @ \mathrm{PCNFs})$ was further investigated by isothermal dehydrogenation and hydrogenation at various temperatures (Figures 6c and d). When heated at $250{ }^{\circ} \mathrm{C}$, only $\sim 2.8 \mathrm{wt} . \% \mathrm{H}_{2}$ could be released from $\mathrm{MgH}_{2} \mathrm{NCs}$ within $200 \mathrm{~min}$, and no hydrogen release was observed for the ball-milled composite, while $\mathrm{MgH}_{2} /(\mathrm{CHNBs} @ \mathrm{PCNFs})$ could desorb 5.9 wt.\% hydrogen within the same period. Moreover, 7.5 wt. \% hydrogen, approaching the theoretical value of $\mathrm{MgH}_{2}$, could be released in less than 25 min when the temperature was increased to $325{ }^{\circ} \mathrm{C}$ (Figure S23). In terms of the reversible hydrogenation, 7.4 wt.\% $\mathrm{H}_{2}$ could be absorbed by $\mathrm{MgH}_{2} /(\mathrm{CHNBs} @ \mathrm{PCNFs})$ within 15 min, even at a temperature as low as $250{ }^{\circ} \mathrm{C}$, while only 3.9 wt. $\% \mathrm{H}_{2}$ was taken up for the $\mathrm{MgH}_{2} \mathrm{NCs}$ 
without the support of CHNBs@PCNFs, and the ball-milled composite exhibited no hydrogenation under the same conditions. More importantly, a complete hydrogenation process, with a capacity of 7.5 wt.\%, was observed for $\mathrm{MgH}_{2} /(\mathrm{CHNBs@PCNFs)}$ in less than 7 min at 325 ${ }^{\circ} \mathrm{C}$. The apparent activation energies $\left(E_{\mathrm{a}}\right)$ for dehydrogenation and hydrogenation of $\mathrm{MgH}_{2} /(\mathrm{CHNBs} @ \mathrm{PCNFs})$ were subsequently calculated according to the Arrhenius equation to quantitatively evaluate the significantly improved dehydriding/hydriding kinetics. After fitting the experimental data at various temperature (Figure S24), the activation energy for dehydrogenation and hydrogenation of $\mathrm{MgH}_{2} /(\mathrm{CHNBs} @ \mathrm{PCNFs})$ was calculated to be 76.4 and $67.1 \mathrm{~kJ} \mathrm{~mol}^{-1}$, respectively, which is significantly lower than the values reported for their bulk counterpart (i.e., $158.5 \mathrm{~kJ} \mathrm{~mol}^{-1}$ for dehydrogenation and $99.0 \mathrm{~kJ} \mathrm{~mol}^{-1}$ for hydrogenation) $)^{[52]}$ and for pure $\mathrm{MgH}_{2} \mathrm{NCs}$ (i.e., $126 \mathrm{~kJ} \mathrm{~mol}^{-1}$ for dehydrogenation) ${ }^{[53]}$ synthesized under the same conditions without the presence of CHNBs@PCNFs. These results obviously confirm the significant improvement of the hydrogenation and dehydrogenation kinetics of $\mathrm{MgH}_{2}$ owing to the catalytic role of $\mathrm{CHNBs@PCNFs,} \mathrm{the} \mathrm{much} \mathrm{shortened} \mathrm{diffusion} \mathrm{pathways} \mathrm{resulting} \mathrm{from} \mathrm{the}$ tremendous reduction in particle size to only $5.1 \mathrm{~nm}$, and the homogenous dispersion of $\mathrm{MgH}_{2}$ NCs on CHNBs@PCNFs, which could further facilitate the catalytic effects of CHNBs@PCNFs.

\section{Conclusions}

In summary, we have demonstrated a novel and general strategy, namely, a scalable and lowcost bubbling-assisted electrospinning method, for producing hierarchical porous carbon nanofibers with a sophisticated structure. By facilely altering the decomposition rate and the amount of bubbling agent, controllable fabrication of carbon hollow nanobubbles on porous carbon nanofibers could be achieved. Benefiting from the excellent electrical conductivity and strong chemical adsorption capability towards NaPSs, CHNBs@PCNFs has been demonstrated 
to be an ideal sulfur host for room-temperature Na-S batteries, while the as-synthesized S/(CHNBs@PCNFs) cathode delivered a high reversible capacity of $913 \mathrm{~mA} \mathrm{~h} \mathrm{~g}^{-1}$ at $0.1 \mathrm{C}$, and exhibited a stable cycle life up to 400 cycles with a small capacity decay of $0.044 \%$ per cycle and a Coulombic efficiency approaching 100\%. When CHNBs@PCNFs are used as a structural support for $\mathrm{MgH}_{2} \mathrm{NCs}$, a complete dehydrogenation at a temperature as low as $250{ }^{\circ} \mathrm{C}$ is realized within only 15 min due to the catalytic role of CHNBs@PCNFs and the homogenous dispersion on them of $\mathrm{MgH}_{2} \mathrm{NCs}$ with a particle size of $5.1 \mathrm{~nm}$. This unique synthetic concept represents a new avenue for the preparation of multifunctional hierarchical porous carbon materials and has great potential for scalable and low-cost industrial production of 1D nanostructured carbon materials.

\section{Experimental details}

Preparation of CHNBs@PCNFs: The raw materials PVA (Mv $\approx 88000$, Alfa Aesar) and $\mathrm{LiN}_{3}$ (20 wt.\%, Alfa Aesar) were commercially available and directly used without further purification. Typically, PVA $(0.5 \mathrm{~g})$ was dissolved in deionized water $(5 \mathrm{~mL})$ at $90{ }^{\circ} \mathrm{C}$ for $8 \mathrm{~h}$. After cooling down to room temperature, $\mathrm{LiN}_{3}$ solution $(0.5 \mathrm{~mL})$ was then added and vigorously stirred for $6 \mathrm{~h}$ make a homogeneous spinning dope. The resultant solution was transferred into a syringe with an 18-gauge blunt-tip needle for electrospinning. The flow rate of solution was

approximately $500 \mu \mathrm{L} \mathrm{h}^{-1}$, as controlled by a syringe pump (Longer, TJP-3A, China), and a grounded stainless steel plate was horizontally placed $10 \mathrm{~cm}$ from the needle to collect the nanowires. A high voltage of $15 \mathrm{kV}$ was applied by a high-voltage power supply. The ascollected electrospun fibers were first dewatered at a temperature of $75{ }^{\circ} \mathrm{C}$ under dynamic vacuum for $15 \mathrm{~h}$ and then calcined to $650{ }^{\circ} \mathrm{C}$ for $3 \mathrm{~h}$ under dynamic $\mathrm{N}_{2}$ atmosphere to obtain the CHNBs@PCNFs after washing with hydrochloric acid and drying. 
Preparation of $\boldsymbol{S}(\boldsymbol{C H N B s @ P C N F s ) : ~ A ~ m i x t u r e ~ o f ~ C H N B s @ P C N F s ~ a n d ~ s u l f u r ~ p o w d e r ~ w i t h ~ a ~}$ weight ratio of 1:4 was sealed in a glass bottle under argon protection, which was then heated at $155^{\circ} \mathrm{C}$ for $12 \mathrm{~h}$, Subsequently, the product was heated at $200{ }^{\circ} \mathrm{C}$ for $30 \mathrm{~min}$ under dynamic argon atmosphere to remove the redundant sulfur outside the CHNBs@PCNFs. The S/CNFs composite was prepared by the same method.

Preparation of $\mathbf{M g H} /\left(\boldsymbol{C H N B s @ P C N F s ) : ~} \mathrm{MgH}_{2} /(\mathrm{CHNBs} @ \mathrm{PCNFs})\left(\mathrm{MgH}_{2}: \mathrm{C}=40: 60\right)\right.$ were synthesized by a wet-chemical method. $1.6 \mathrm{ml}$ di-n-butylmagnesium solution in heptane was added to $40 \mathrm{ml}$ cyclohexane in a pressure reactor vessel. The solution was then stirred and heated to $200{ }^{\circ} \mathrm{C}$ under $3.5 \mathrm{MPa}$ hydrogen pressure. After $24 \mathrm{~h}$, the $\mathrm{MgH}_{2} /(\mathrm{CHNBs} @ \mathrm{PCNFs})$ was obtained from the decomposition of di-n-butylmagnesium in cyclohexane. The precipitates were washed several times with cyclohexane. The obtained materials were further dried at room temperature under dynamic vacuum on a Schlenk line.

Adsorption test: Sodium polysulfide $\left(\mathrm{Na}_{2} \mathrm{~S}_{8}\right)$ was prepared by mixing $7 \mathrm{~mol}$ of $\mathrm{S}$ with $1 \mathrm{~mol}$ of $\mathrm{Na}_{2} \mathrm{~S}$ in tetraethylene glycol dimethyl ether (TEGDME) solvent. After mechanical stirring at 40 ${ }^{\circ} \mathrm{C}$ for $24 \mathrm{~h}$ in an $\mathrm{Ar}$-filled glove box, a brownish-red $\mathrm{Na}_{2} \mathrm{~S}_{8}$ solution was synthesized, which was then diluted to $1.25 \mathrm{mM}$ for the polysulfide adsorption test. The adsorption test was conducted by immersing $15 \mathrm{mg}$ of the as-synthesized CNFs and CHNBs@PCNFs in $2 \mathrm{~mL}$ of $0.005 \mathrm{M}$ $\mathrm{Na}_{2} \mathrm{~S}_{8}$ solution for $30 \mathrm{~min}$. Digital photographs were taken after the solution was left to stand for $30 \min$.

Characterizations: The crystalline structure of the samples was characterized by X-ray diffraction (XRD) conducted on a Rigaku D/max-kA diffractometer with $\mathrm{Cu} \mathrm{K} \alpha$ radiation. (An amorphous tape was used to prevent the oxidation and moisture contamination of samples during 
XRD measurements.) Nitrogen absorption/desorption isotherms (Brunauer-Emmett-Teller (BET) technique) at the temperature of liquid nitrogen were collected via a Quantachrome NOVA 4200e instrument to characterize the pore structure of the samples. Thermal property measurements were performed by thermogravimetry (TG, STA 409C) with a heating rate of $5{ }^{\circ} \mathrm{C}$ $\min ^{-1}$ under Ar. The microstructures were observed by transmission electron microscopy (TEM, JEM-2100F, JEOL) and field-emission scanning electron microscopy (SEM, S-4800, Hitachi). The X-ray photoelectron spectroscopy (XPS) was conducted on a Perkin Elmer PHI 5000C ESCA system equipped with a dual X-ray source, in which an $\mathrm{Mg} \mathrm{K \alpha}(1253.6 \mathrm{eV})$ anode and a hemispherical energy analyser were used. The background pressure during data acquisition was maintained at $<10^{-6} \mathrm{~Pa}$, and measurements were conducted at a pass energy of $93.90 \mathrm{eV}$. All binding energies were calibrated using contaminant carbon $(\mathrm{C} 1 \mathrm{~s}=284.6 \mathrm{eV})$.

Electrochemical measurements: CR2032 coin cells were assembled with sodium metal as the counter and reference electrodes in an argon-filled glove box. The S/CHNBs@PCNFs and S/CNFs cathode slurries were made by mixing $80 \mathrm{wt} \%$ active materials with $10 \mathrm{wt} \%$ carbon black and $10 \mathrm{wt} \%$ carboxyl methylated cellulose (CMC) in water by mortar and pestle. The resultant slurry was then casted on the aluminium foil using a doctor blade prior to drying in a vacuum oven at $60{ }^{\circ} \mathrm{C}$ for $20 \mathrm{~h}$. The areal mass loading of sulfur in this study is controlled to be around $1.4 \mathrm{mg} \mathrm{cm}^{-2}$. Glass fiber purchased from Whatman was used as the separator. The electrolyte used was $1 \mathrm{M}$ sodium perchlorate $\left(\mathrm{NaClO}_{4}\right)$ in ethylene carbonate/propylene carbonate (EC/PC, 1:1 v/v\%) with the addition of $5 \mathrm{wt} . \%$ fluoroethylene carbonate (FEC). The assembled cells were galvanostatically charged and discharged over the voltage range of $1.2-$ $2.8 \mathrm{~V}$ versus $\mathrm{Na} / \mathrm{Na}^{+}$at various constant current densities on a Land CT-2001A battery tester. 
Electrochemical impedance spectroscopy and cyclic voltammetry were performed on a VMP-3 electrochemical workstation at a scan rate of $0.1 \mathrm{mV} \mathrm{s}^{-1}$.

Hydrogen storage measurements: The hydrogen storage properties of the thus-synthesized $\mathrm{MgH}_{2} /(\mathrm{CHNBs} @ \mathrm{PCNFs})$ were investigated on a Sieverts apparatus, described as a gas reaction controller (GRC, Advanced Materials Corp., USA). The hydriding kinetics measurements were performed with an initial pressure of $30 \mathrm{~atm}$, and the dehydriding properties were detected at various temperatures under a hydrogen pressure below 0.02 atm. For the purposes of comparison, CHNBs@PCNFs were excluded when determining the capacity of hydrogen from the relevant composites.

Supporting Information Available: Supplementary data associated with this article can be found in the online version at xxx.

\section{Acknowledgements}

This work was partially supported by the National Key Research and Development Program of China (2017YFA0204600), National Science Fund for Distinguished Young Scholars (51625102), the National Natural Science Foundation of China (51471053), and the Science and Technology Commission of Shanghai Municipality (17XD1400700). G. Xia is grateful for the finacial support from ARC (DE170100362). The authors also would like to thank Dr. Tania Silver for critical reading of the manuscript.

\section{References}


[1] Y. Sun, N. Liu, Y. Cui, Nature Energy 2016, 1, 16071.

[2] B. Dunn, H. Kamath, J.-M. Tarascon, Science 2011, 334, 928.

[3] S.-i. Orimo, Y. Nakamori, J. R. Eliseo, A. Züttel, C. M. Jensen, Chemical Reviews 2007, $107,4111$.

[4] P. Chen, M. Zhu, Materials Today 2008, 11, 36.

[5] L. Schlapbach, A. Zuttel, Nature 2001, 414, 353.

[6] L. Schlapbach, Nature 2009, 460, 809.

[7] A. Manthiram, Y. Fu, S.-H. Chung, C. Zu, Y.-S. Su, Chemical Reviews 2014, 114, 11751.

[8] M. Wild, L. O'Neill, T. Zhang, R. Purkayastha, G. Minton, M. Marinescu, G. J. Offer, Energy \& Environmental Science 2015, 8, 3477.

[9] A. Manthiram, X. Yu, Small 2015, 11, 2108.

[10] S. Wei, S. Xu, A. Agrawral, S. Choudhury, Y. Lu, Z. Tu, L. Ma, L. A. Archer, Nature Communications 2016, 7, 11722.

[11] X. Lu, G. Xia, J. P. Lemmon, Z. Yang, Journal of Power Sources 2010, 195, 2431.

[12] Z. Wen, Y. Hu, X. Wu, J. Han, Z. Gu, Advanced Functional Materials 2013, 23, 1005.

[13] T. H. Hwang, D. S. Jung, J.-S. Kim, B. G. Kim, J. W. Choi, Nano Letters 2013, 13, 4532.

[14] S. Wenzel, H. Metelmann, C. Raiß, A. K. Dürr, J. Janek, P. Adelhelm, Journal of Power Sources 2013, 243, 758.

[15] S. Xin, Y.-X. Yin, Y.-G. Guo, L.-J. Wan, Advanced Materials 2014, 26, 1261.

[16] X. Yu, A. Manthiram, Advanced Energy Materials 2015, 5, 1500350.

[17] X. Yu, A. Manthiram, ChemElectroChem 2014, 1, 1275.

[18] X. Yu, A. Manthiram, The Journal of Physical Chemistry C 2014, 118, 22952. 
[19] X. Yu, A. Manthiram, The Journal of Physical Chemistry Letters 2014, 5, 1943.

[20] Z. Qiang, Y.-M. Chen, Y. Xia, W. Liang, Y. Zhu, B. D. Vogt, Nano Energy 2017, 32, 59.

[21] R. Carter, L. Oakes, A. Douglas, N. Muralidharan, A. P. Cohn, C. L. Pint, Nano Letters 2017, 17, 1863.

[22] L. Zeng, Y. Yao, J. Shi, Y. Jiang, W. Li, L. Gu, Y. Yu, Energy Storage Materials 2016, 5,50 .

[23] Y.-X. Wang, J. Yang, W. Lai, S.-L. Chou, Q.-F. Gu, H. K. Liu, D. Zhao, S. X. Dou, Journal of the American Chemical Society 2016, 138, 16576.

[24] I. Kim, C. H. Kim, S. h. Choi, J.-P. Ahn, J.-H. Ahn, K.-W. Kim, E. J. Cairns, H.-J. Ahn, Journal of Power Sources 2016, 307, 31.

[25] Y.-X. Yin, S. Xin, Y.-G. Guo, L.-J. Wan, Angewandte Chemie International Edition 2013, 52, 13186.

[26] Z. Li, J. T. Zhang, Y. M. Chen, J. Li, X. W. Lou, Nature Communications 2015, 6, 8850.

[27] H. B. Wu, G. Zhang, L. Yu, X. W. Lou, Nanoscale Horizons 2016, 1, 27.

[28] Z. Li, H. B. Wu, X. W. Lou, Energy \& Environmental Science 2016, 9, 3061.

[29] J. S. Lee, J. Jun, J. Jang, A. Manthiram, Small 2017, 13, 1602984.

[30] K. Mi, Y. Jiang, J. Feng, Y. Qian, S. Xiong, Advanced Functional Materials 2016, 26, 1571.

[31] G. Xia, D. Li, X. Chen, Y. Tan, Z. Tang, Z. Guo, H. Liu, Z. Liu, X. Yu, Advanced Materials 2013, 25, 6238 .

[32] Y. Liu, N. Zhang, L. Jiao, J. Chen, Advanced Materials 2015, 27, 6702.

[33] G. Xia, L. Zhang, F. Fang, D. Sun, Z. Guo, H. Liu, X. Yu, Advanced Functional Materials 2016, 26, 6188 . 
[34] J. Song, Z. Yu, M. L. Gordin, D. Wang, Nano Letters 2016, 16, 864.

[35] G. Zhou, E. Paek, G. S. Hwang, A. Manthiram, Advanced Energy Materials 2016, 6, 1501355.

[36] J. Song, M. L. Gordin, T. Xu, S. Chen, Z. Yu, H. Sohn, J. Lu, Y. Ren, Y. Duan, D. Wang, Angewandte Chemie International Edition 2015, 54, 4325.

[37] X. Yu, A. Manthiram, Chemistry of Materials 2016, 28, 896.

[38] G. Zhou, Y. Zhao, C. Zu, A. Manthiram, Nano Energy 2015, 12, 240.

[39] G. Zhou, Y. Zhao, A. Manthiram, Advanced Energy Materials 2015, 5, 1402263.

[40] G. Zhou, E. Paek, G. S. Hwang, A. Manthiram, Nature Communications 2015, 6, 7760.

[41] K.-F. Aguey-Zinsou, J.-R. Ares-Fernandez, Energy \& Environmental Science 2010, 3, 526.

[42] H. Shao, G. Xin, J. Zheng, X. Li, E. Akiba, Nano Energy 2012, 1, 590.

[43] P. E. de Jongh, T. M. Eggenhuisen, Advanced Materials 2013, 25, 6672.s

[44] T. K. Nielsen, F. Besenbacher, T. R. Jensen, Nanoscale 2011, 3, 2086.

[45] M. L. Christian, K.-F. Aguey-Zinsou, ACS Nano 2012, 6, 7739.

[46] G. Xia, Y. Tan, X. Chen, D. Sun, Z. Guo, H. Liu, L. Ouyang, M. Zhu, X. Yu, Advanced Materials 2015, 27, 5981 .

[47] G. Liu, Y. Wang, L. Jiao, H. Yuan, ACS Applied Materials \& Interfaces 2014, 6, 11038.

[48] W. Cai, X. Zhou, L. Xia, K. Jiang, S. Peng, X. Long, J. Liang, Journal of Materials Chemistry A 2014, 2, 16369.

[49] Y. Jia, X. Yao, International Journal of Hydrogen Energy 2017, 42, 22933.

[50] Y. Liu, J. Zou, X. Zeng, W. Ding, RSC Advances 2014, 4, 42764. 
[51] Y. S. Au, M. K. Obbink, S. Srinivasan, P. C. M. M. Magusin, K. P. de Jong, P. E. de Jongh, Advanced Functional Materials 2014, 24, 3604.

[52] G. Liu, Y. Wang, C. Xu, F. Qiu, C. An, L. Li, L. Jiao, H. Yuan, Nanoscale 2013, 5, 1074.

[53] N. S. Norberg, T. S. Arthur, S. J. Fredrick, A. L. Prieto, Journal of the American

Chemical Society 2011, 133, 10679. 

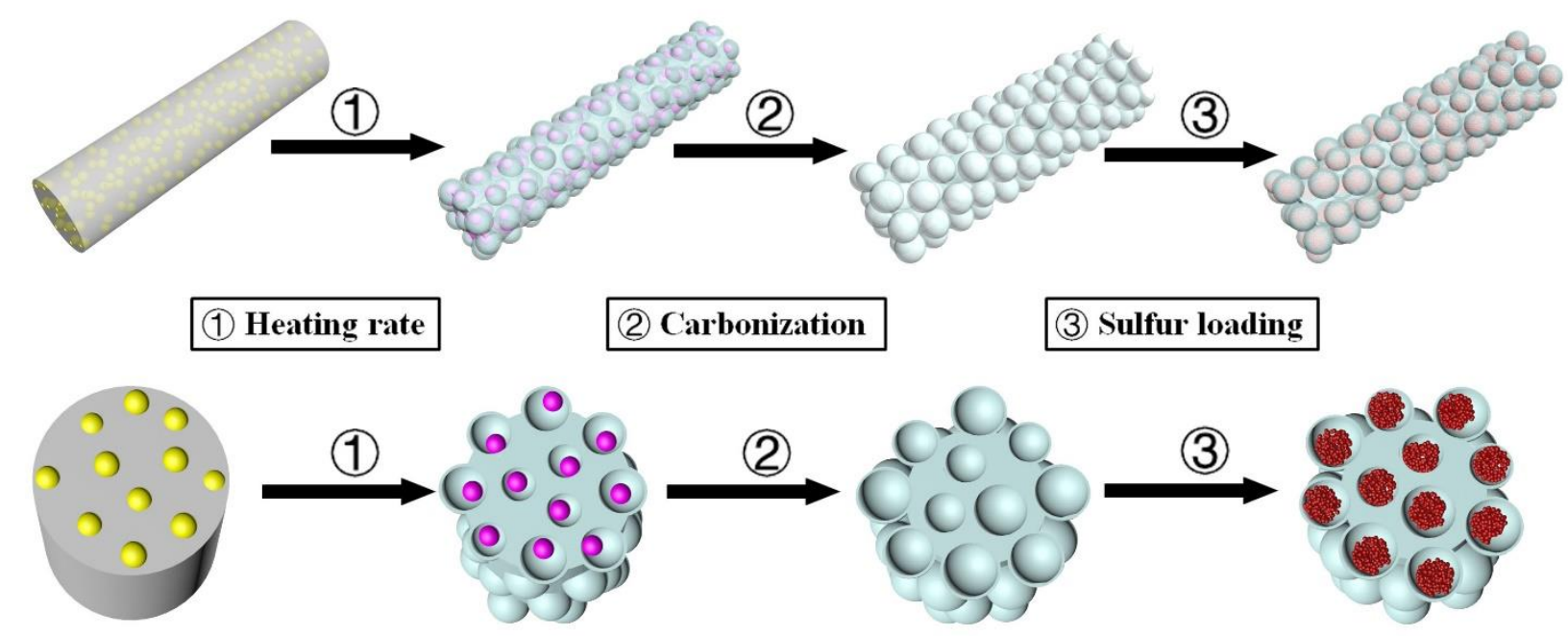

Figure 1. Schematic illustration of the preparation of CHNBs@PCNFs and their application as sulfur hosts for room temperature Na-S batteries. Yellow, pink, and red balls represent $\mathrm{LiN}_{3}$, $\mathrm{Li}_{3} \mathrm{~N}$, and $\mathrm{S}$ particles, respectively. 

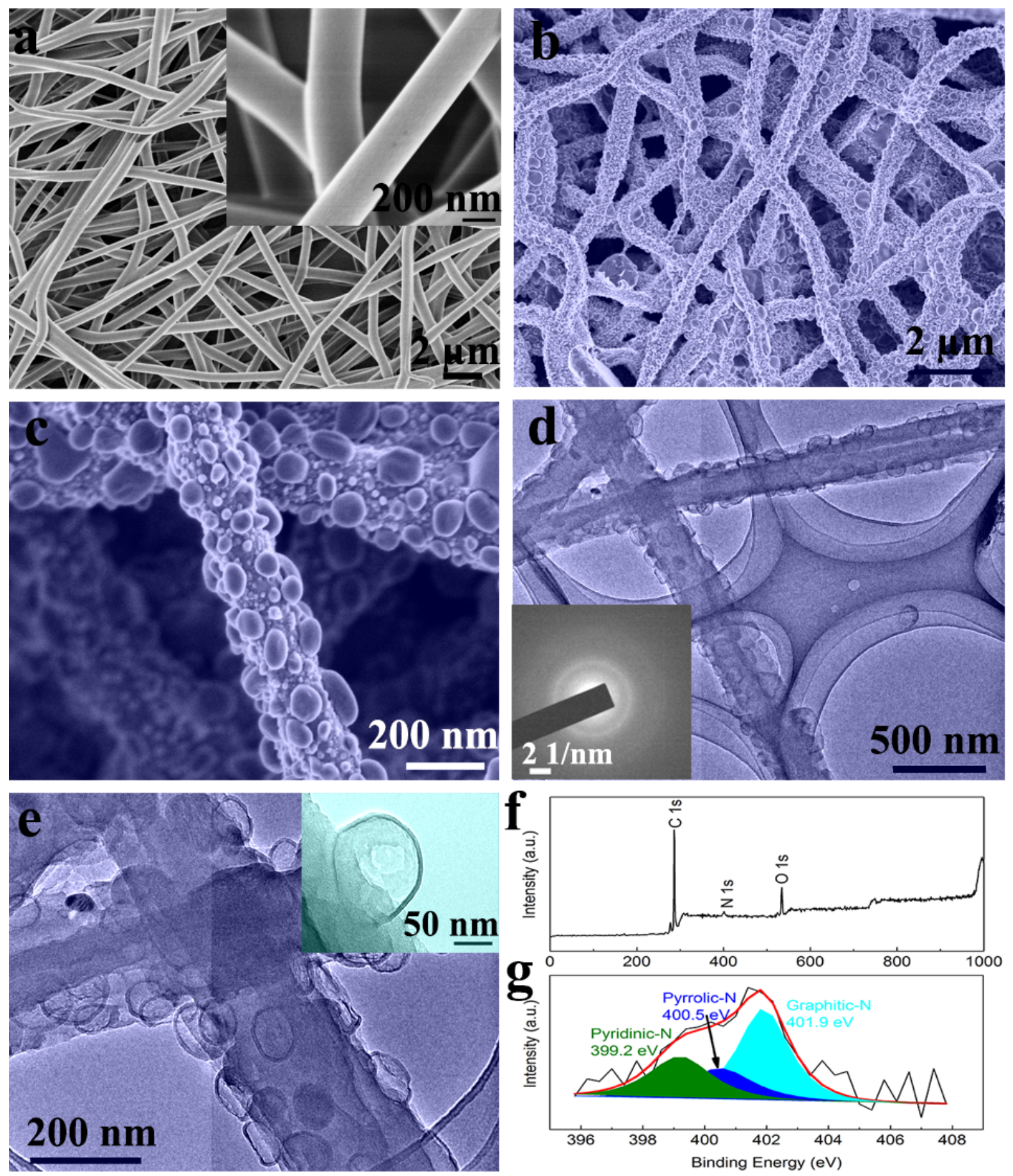

Figure 2. (a) SEM image of the as-electrospun PVA-LiN $\mathrm{L}_{3}$ nanofibers. The inset of (a) shows a magnified image of as-electrospun PVA nanofibers. (b, c) SEM images of PCNFs after calcination of as-electrospun PVA-LiN 3 nanofibers. (d, e) TEM images of the as-synthesized CHNBs@PCNFs at different magnifications. The inset of (d) and (e) shows the SAED patterns of CHNBs@PCNFs and a magnified image of CHNBs@PCNFs, respectively. XPS survey spectrum (f) and high-resolution N 1s spectrum (g) of CHNBs@PCNFs. 

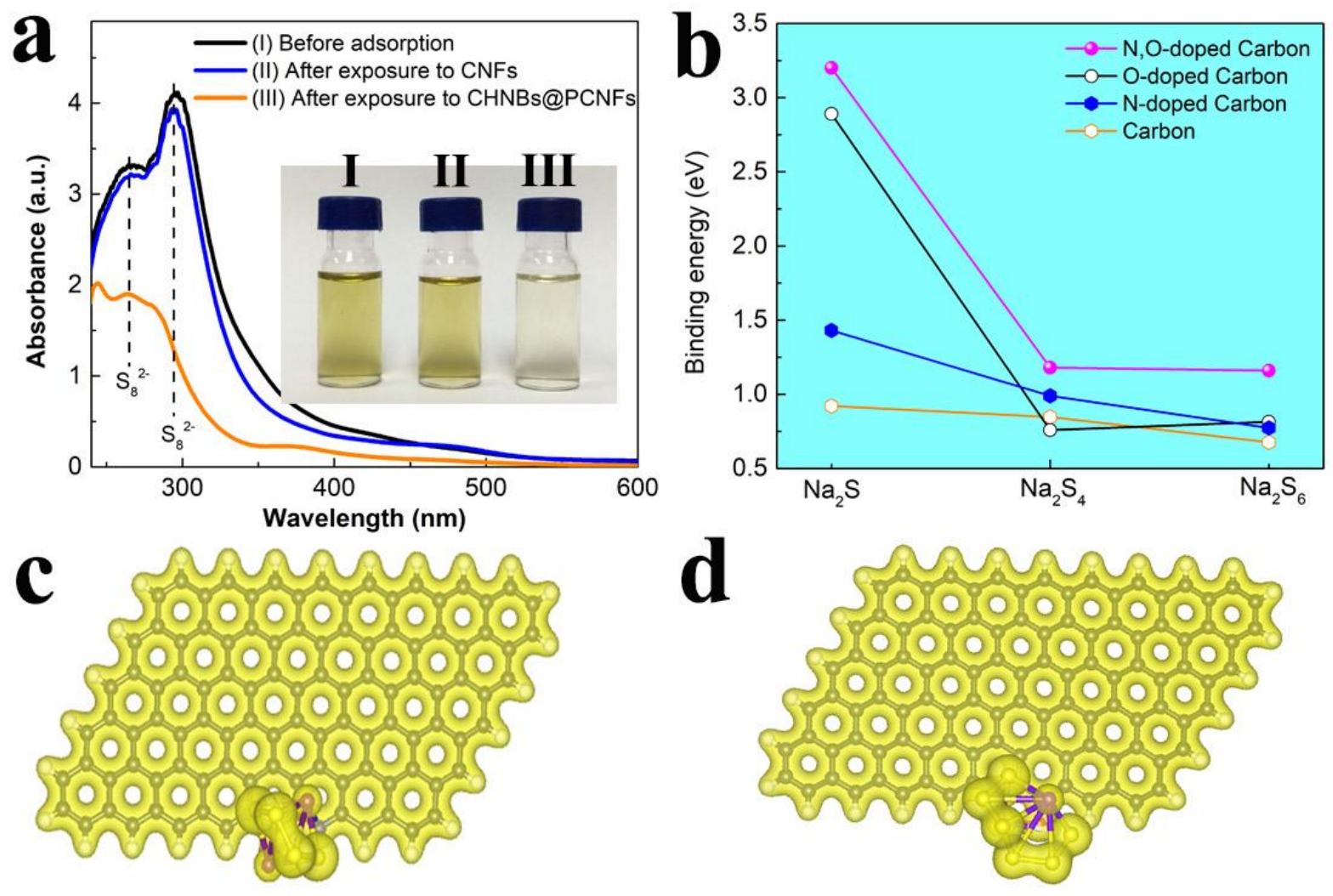

d
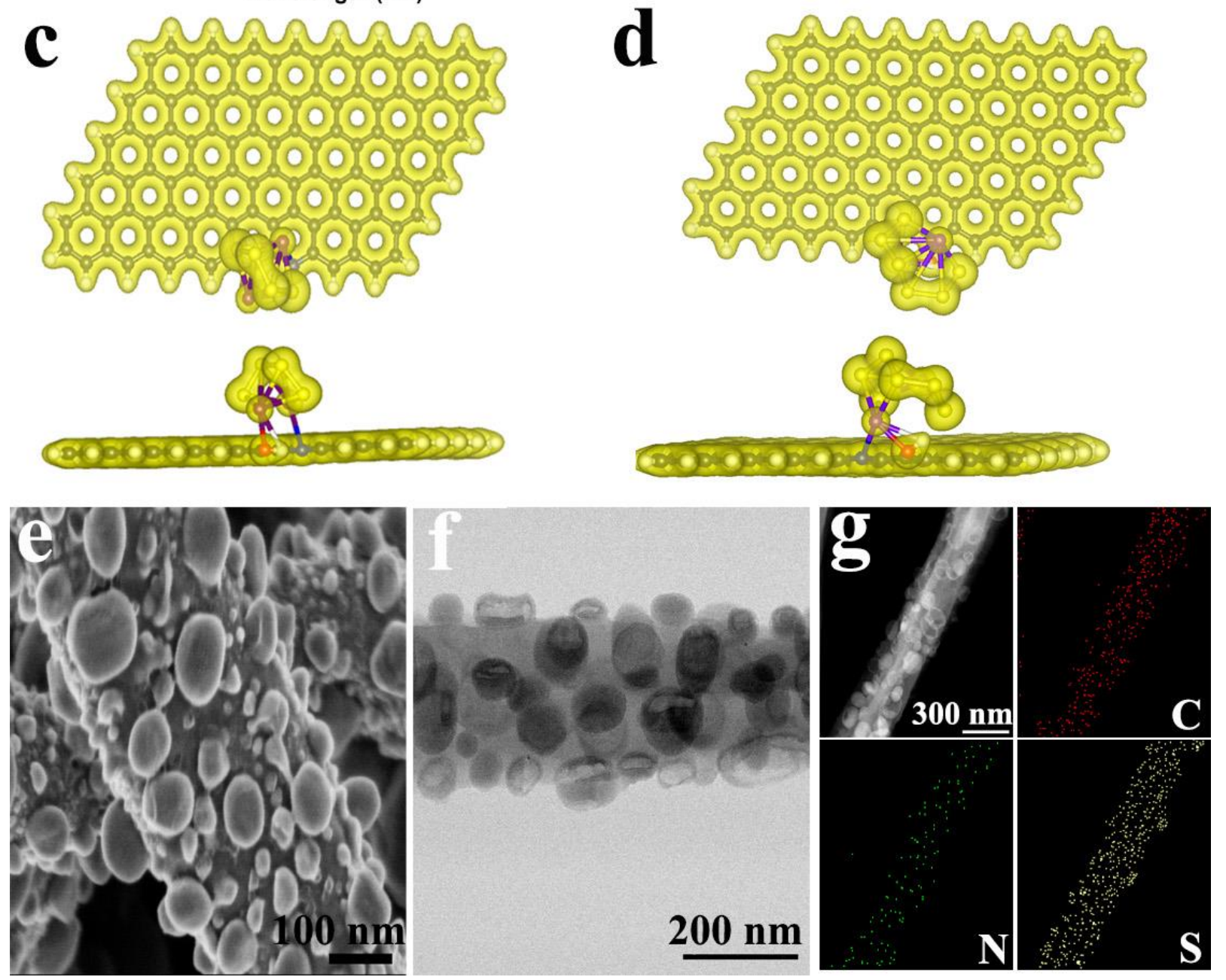

Figure 3. (a) UV-vis spectra and corresponding photograph (inset) of the pure NaPSs solution (I) and the solution after exposure to the different substances: (II) CNFs, and (III) CHNBs@PCNFs 
(photographs taken after the solutions were allowed to stand for $30 \mathrm{~min}$ ). (b) Comparison of the binding energy of various NaPSs molecules binding to the pure carbon, O-doped carbon, Ndoped carbon, and N, O-codoped carbon, respectively. Atom positions and charge density plot of $\mathrm{Na}_{2} \mathrm{~S}_{4}$ (c) and $\mathrm{Na}_{2} \mathrm{~S}_{6}$ (d) molecular interaction with N, O-codoped carbon. Grey, white, blue, yellow, purple, and red balls represent $\mathrm{C}, \mathrm{H}, \mathrm{N}, \mathrm{S}, \mathrm{Na}$, and $\mathrm{O}$ atoms, respectively. (e) SEM and (f) TEM images of S/(CHNBs@PCNFs). (g) Scanning (STEM) image and the corresponding elemental mapping of $\mathrm{S} /(\mathrm{CHNBs} @ \mathrm{PCNFs})$.
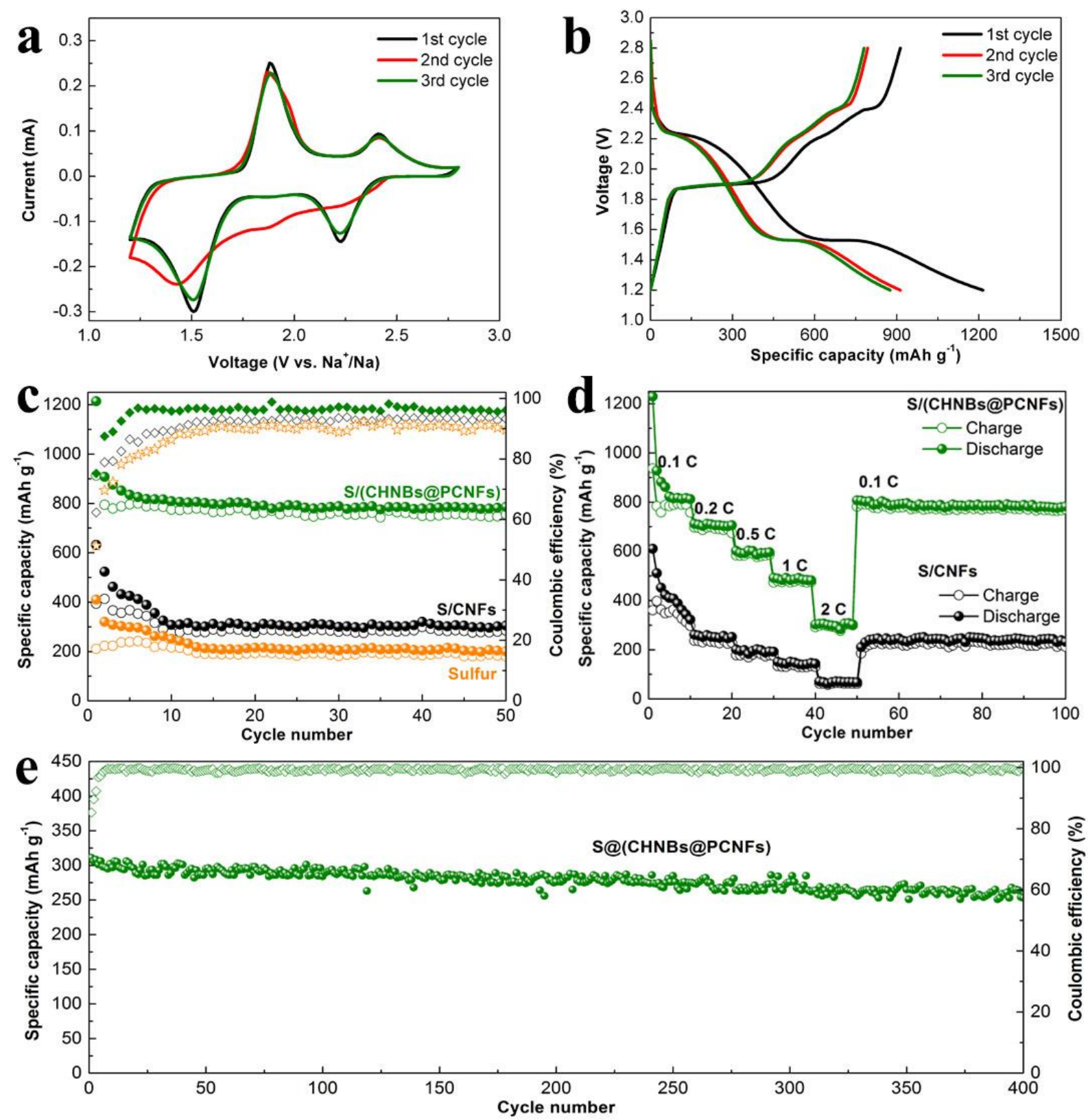

Figure 4. Electrochemical performance of the S/(CHNBs@PCNFs) electrode in Na-S batteries. (a) Cyclic voltammograms for the initial three cycles of the S/(CHNBs@PCNFs) electrode at a 
scanning rate of $0.2 \mathrm{mV} \mathrm{s}^{-1}$. (b) Galvanostatic discharge-charge profiles for the first 3 cyles at a current density of $0.1 \mathrm{C}$. (c) Cycling performance at a current density of $0.1 \mathrm{C}$ and coulombic efficiency of S/(CHNBs@PCNFs) electrode, with S@CNFs included for comparison. (d) Rate performance at different current densities from 0.1 C to $2 \mathrm{C}$, with S@CNFs and pure sulfur included for comparison. (e) Cycling performance at a current density of $2 \mathrm{C}$.

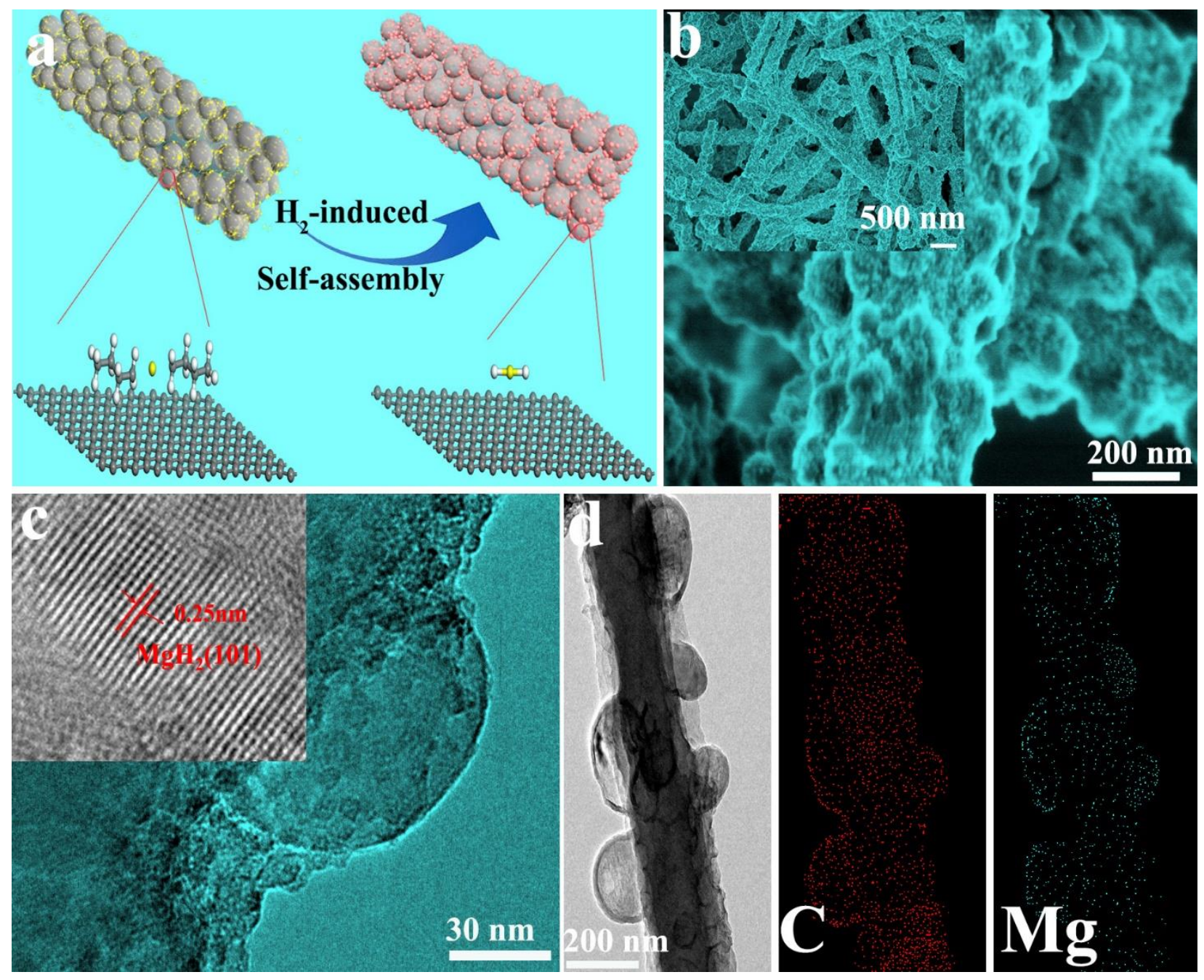

Figure 5. (a) Schematic illustration of the preparation of $\mathrm{MgH}_{2} /(\mathrm{CHNBs} @ \mathrm{PCNFs})$ through the hydrogenation-induced self-assembly strategy. Grey, white, and yellow spheres are $\mathrm{C}, \mathrm{H}$, and $\mathrm{Mg}$ atoms, respectively. (b) SEM and (c) TEM images of $\mathrm{MgH}_{2} /(\mathrm{CHNBs@PCNFs)} \mathrm{under}$ different magnifications (with the inset of c showing an HRTEM image). (d) TEM image and the corresponding elemental mapping of $\mathrm{MgH}_{2} /(\mathrm{CHNBs} @ \mathrm{PCNFs})$. 

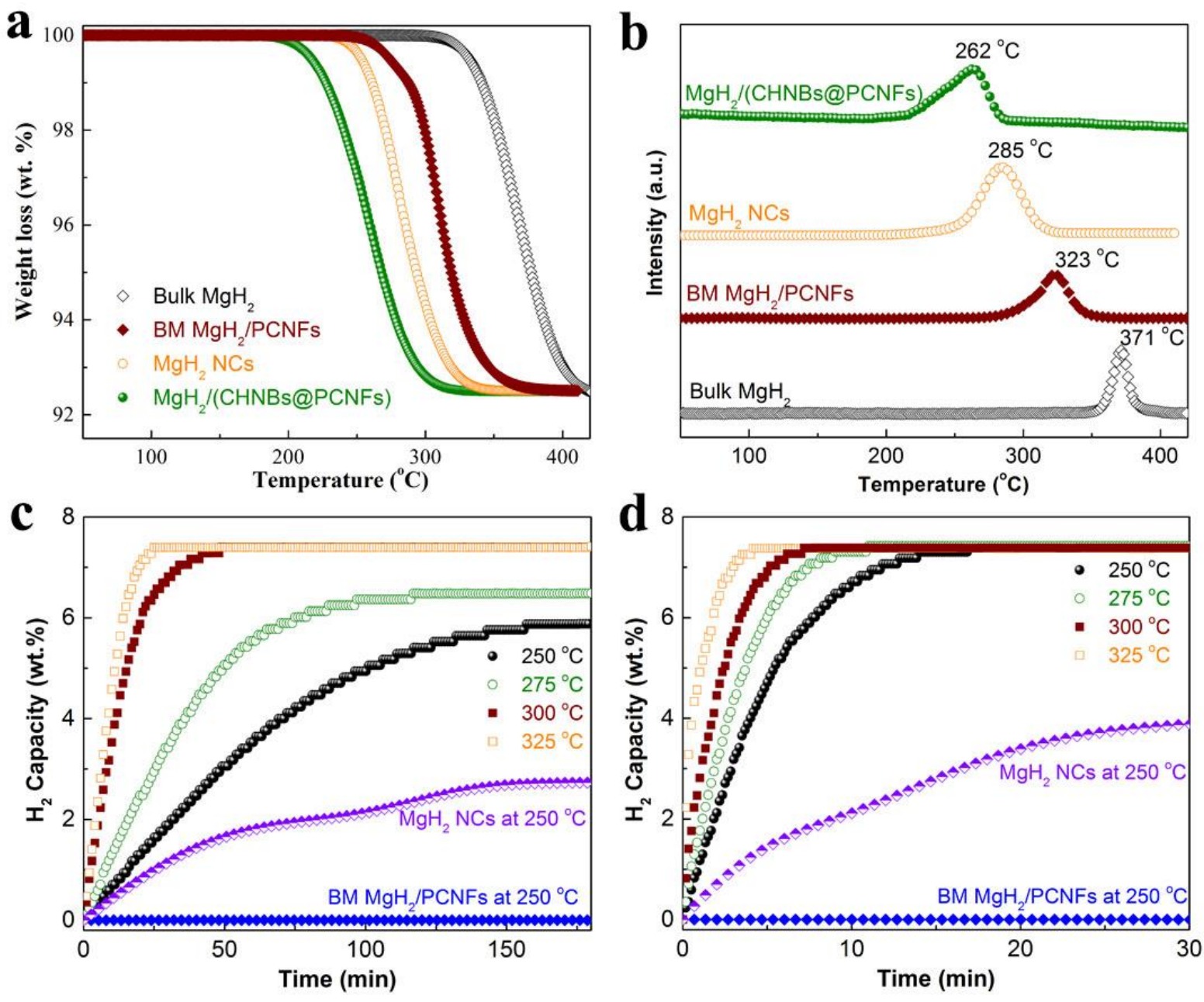

Figure 6. TGA (a) and MS (b) profiles of $\mathrm{MgH}_{2} /\left(\mathrm{CHNBs@PCNFs),} \mathrm{with} \mathrm{bulk} \mathrm{MgH}_{2}\right.$, a ballmilled (BM) composite of $\mathrm{MgH}_{2}$ and CHNBs@PCNFs (BM MgH $/ \mathrm{PCNFs}$ ), and $\mathrm{MgH}_{2} \mathrm{NCs}$ included for comparison. Isothermal dehydrogenation (c) and hydrogenation (d) profiles of $\mathrm{MgH}_{2} /(\mathrm{CHNBs} @ \mathrm{PCNFs})$ at different temperatures. 